\title{
A mathematical model for peristaltic transport of micro-polar fluids
}

\author{
S.K. Pandey and Dharmendra Tripathi* \\ Department of Applied Mathematics, Institute of Technology, Banaras Hindu University, Varanasi 221005, India
}

\begin{abstract}
A mathematical model has been constructed for peristaltic transport of micro-polar fluid in a circular cylindrical tube of finite length by letting sinusoidal waves propagate along the wall that induce contraction and relaxation but not expansion beyond the natural boundary. Axial and radial velocities and micro-rotation components are formulated for micro-polar fluid transportations by applying the method of long wavelength and low Reynolds number approximations in the analysis. Pressure distribution along the tube length is studied to investigate temporal effects. An in-depth study has been done to learn the effects of coupling number and micro-polar parameter. The effects of coupling number and micro-polar parameter are investigated also on mechanical efficiency, reflux and trapping. A significant difference observed is that unlike integral wave-trains propagating along the tube walls that have identical peaks of pressure, non-integral wave-trains have peaks of different sizes.
\end{abstract}

Keywords: Peristaltic transport, oesophagus, micro-polar fluid, mechanical efficiency, reflux, trapping

\section{Introduction}

Taking into consideration the effects of individual particles such as micro-rotation in the flow, a concentrated suspension of non-deformable neutrally buoyant rigid particles in a viscous medium was formulated by Eringen [1]. For the reason that the effects are microrotational and micro-inertial, such a fluid was named micro-polar.

Micro-polar fluids are very common type of fluids such as blood, chyme, some edible solutions, polymer solutions, colloidal solutions, drilling fluids in oil industries. Some food materials such as solutions of roasted cereal powders consumed in Indian subcontinent may be viewed as micro-polar fluid.

Devi and Devanathan [2] investigated the peristaltic transport of micro-polar fluid in a cylindrical tube with a sinusoidal wave of small amplitude. Using long wavelength approximations, Philip and Chandra [3] worked on the peristaltic transport of a simple

*Corresponding author. Dharmendra Tripathi, Department of Applied Mathematics, Institute of Technology, Banaras Hindu University, Varanasi-221005, India. E-mail: dtripathi.rs.apm@ itbhu.ac.in. micro-polar fluid which accounts for micro-rotation and micro-stretching of the particles contained in a small volume element.

Recently, Bhargava et al. [4] modelled the peristaltic flow of blood through stenosed arteries, by considering blood as micro-polar fluid and arteries as porous channel. Srinivasacharya et al. [5] studied different micro-polar properties on pressure across one wavelength and trapping; Hayat et al. [6] investigated the effects of different wave forms; Muthu et al. [7, 8] studied wall properties in channels and tubes respectively whereas Hayat and Ali [9] reported effects of an endoscope. The same authors [10] studied the effects of asymmetricity of wave propagation in a channel while Mekheimer and Elmaboud [11] studied the flow in an annulus.

All the researchers, cited above, applied long wavelength and low Reynolds number approximations on an infinite tube with the walls contracting and dilating as well to the same extent. The fluid was considered as micro-polar. In none of those studies, the inherent phenomenon reflux and mechanical efficiency have found place anywhere in discussion. The studies are confined to the propagation of only integral number of 
waves. Moreover, investigations at micro-level such as pressure at each point along the entire length of vessel that we intend to carry out with regard to pressure distribution have been ignored.

However, the fact is that the physiological vessels, in general, and oesophagus, in particular, undergo only contraction followed by relaxation, but no expansion beyond the natural boundary. Oesophagus is an 18 to $26 \mathrm{~cm}$ long and 1.5 to $2.5 \mathrm{~cm}$ wide collapsible muscular tube stretching from the upper sphincter to the lower sphincter. Swallowing has hardly anything to do with gravitational force on the fluid because even if one is upside down, swallowing will take place. Cattle grazing in the field have their neck down toward the ground; yet they swallow easily.

This is revealed from the aforementioned discussion that the oesophagus is finite in length in comparison to its radius. The consideration of wall's expansion beyond the stationary boundary not only makes a vital difference of qualitative nature but also a quantitative difference is expected. Hence, none of the studies mentioned above is suitable for describing swallowing of a micro-polar fluid in oesophagus.

Dodds [12] and Ren et al. [13] carried out experimental investigations in this direction for different viscosities and bolus-volumes. Li and Brasseur [14] have done theoretical investigation on it for Newtonian and reported similar aspects of various non-Newtonian fluids.

We intend to investigate swallowing of a nonNewtonian foodstuff of micro-polar nature through oesophagus by assuming it a cylindrical tube of finite length. This model may fit to blood flow in aorta and flow of semi-digested food in duodenum. Propagation of a non-integral number of waves along the tube walls, which is an inherent characteristic of finite length vessels, can also be investigated.

\section{Mathematical model}

We consider the flow of a micro-polar fluid in a tube of length $\tilde{l}$ caused by continuous contraction waves that propagate on the walls of the tube (cf. Fig. 1) and are given by

$$
\tilde{h}(\tilde{x}, \tilde{t})=a-\tilde{\phi} \cos ^{2} \frac{\pi}{\lambda}(\tilde{x}-c \tilde{t}),
$$

where $\tilde{h}, \tilde{x}, \tilde{t}, a, \tilde{\phi}, \lambda$ and $c$ respectively stand for radial displacement of the wall, axial coordinate, time, radius of the tube, amplitude of the wave, wavelength and wave velocity.

The governing equations of the flow of micro-polar fluid in the absence of body forces and body couple are given by

$$
\begin{array}{r}
\rho\left(\frac{\partial \tilde{u}}{\partial \tilde{t}}+\tilde{u} \frac{\partial \tilde{u}}{\partial \tilde{x}}+\tilde{v} \frac{\partial \tilde{u}}{\partial \tilde{r}}\right)=-\frac{\partial \tilde{p}}{\partial \tilde{x}}+k \frac{1}{\tilde{r}} \frac{\partial(\tilde{r} \tilde{w})}{\partial \tilde{r}}+(\mu+k)\left(\frac{\partial^{2} \tilde{u}}{\partial \tilde{x}^{2}}+\frac{1}{\tilde{r}} \frac{\partial}{\partial \tilde{r}}\left(\tilde{r} \frac{\partial \tilde{u}}{\partial \tilde{r}}\right)\right), \\
\rho\left(\frac{\partial \tilde{v}}{\partial \tilde{t}}+\tilde{u} \frac{\partial \tilde{v}}{\partial \tilde{x}}+\tilde{v} \frac{\partial \tilde{v}}{\partial \tilde{r}}\right)=-\frac{\partial \tilde{p}}{\partial \tilde{r}}-k \frac{\partial \tilde{w}}{\partial \tilde{x}}+(\mu+k)\left(\frac{\partial^{2} \tilde{v}}{\partial \tilde{x}^{2}}+\frac{\partial}{\partial \tilde{r}}\left(\frac{1}{\tilde{r}} \frac{\partial \tilde{v}}{\partial \tilde{r}}\right)\right), \\
\rho \tilde{\sigma}\left(\frac{\partial \tilde{w}}{\partial \tilde{t}}+\tilde{u} \frac{\partial \tilde{w}}{\partial \tilde{x}}+\tilde{v} \frac{\partial \tilde{w}}{\partial \tilde{r}}\right)=-2 k \tilde{w}+k\left(\frac{\partial \tilde{v}}{\partial \tilde{x}}-\frac{\partial \tilde{u}}{\partial \tilde{r}}\right)+\gamma\left(\frac{\partial^{2} \tilde{w}}{\partial \tilde{x}^{2}}+\frac{\partial}{\partial \tilde{r}}\left(\frac{1}{\tilde{r}} \frac{\partial \tilde{w}}{\partial \tilde{r}}\right)\right)+(\alpha+\beta+\gamma) \tilde{\nabla}(\tilde{\nabla} \cdot \tilde{w}),
\end{array}
$$

fluids considering finite length tubes, and focussed the study on both the local and global dynamics. The issue of local dynamics such as spatial-temporal variations in local stresses in terms of the motility and efficacy of the transport process was raised by Brasseur and Dodds [15]. They found close resemblance with the experimental findings of Dodds [12] and Ren et al. [13]. Misra and Pandey [16], who investigated similar phenomena for power-law fluids, reported similar conclusions, although they didn't discuss the experimental findings. Pandey and Tripathi [17-20] have worked out

$$
\frac{\partial \tilde{u}}{\partial \tilde{x}}+\frac{1}{\tilde{r}} \frac{\partial(\tilde{r} \tilde{v})}{\partial \tilde{r}}=0
$$

where $\tilde{u}, \tilde{v}, \tilde{w}, \tilde{r}, \rho, \tilde{\sigma}$ are axial velocity, radial velocity, micro-polar vector, radial coordinate, fluid density, micro-gyration parameter, respectively and $\mu, k, \alpha$, $\beta, \gamma$ are material constants and satisfy the following conditions:

$$
\begin{array}{r}
2 \mu+k \geq 0, \quad k \geq 0, \quad 3 \alpha+\beta+\gamma \geq 0, \\
\gamma \geq|\beta| .
\end{array}
$$




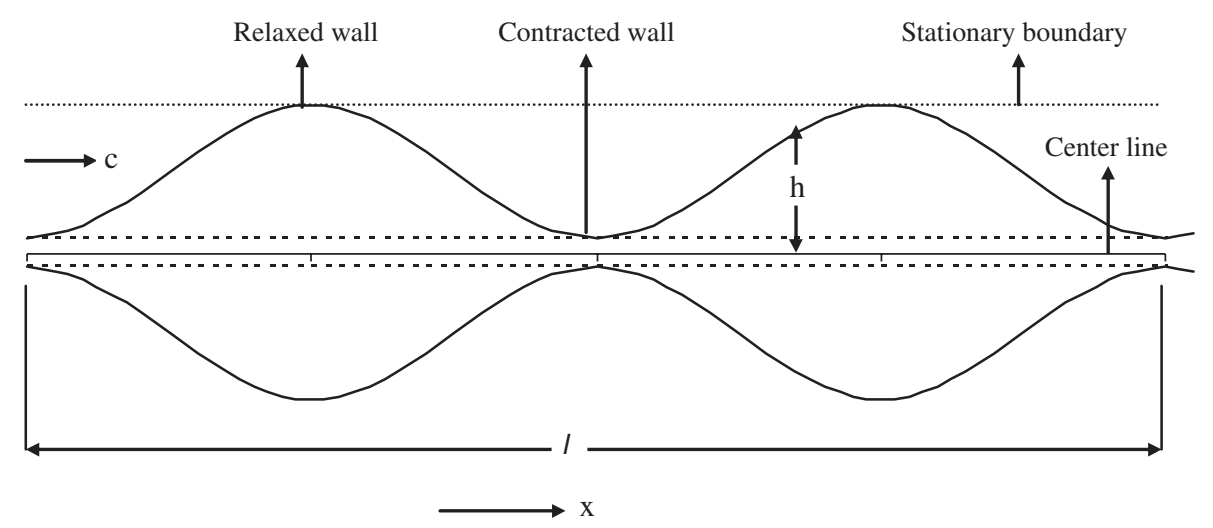

Fig. 1. The diagram, based on Eq. (12), represents the propagation of a progressive wave along the walls of the tube containing fluid, which undergoes contraction and relaxation but no expansion beyond the boundary. $h$ is the radial displacement of the wall, $c$ is the wave velocity, $l$ is the length of tube and $x$ is the axial coordinate.

For the subsequent analysis, the following nondimensional parameters are introduced:
In view of non-dimensionalisation, Eq. (1) takes the following dimensionless form:

$$
\begin{aligned}
& x=\frac{\tilde{x}}{\lambda}, r=\frac{\tilde{r}}{a}, t=\frac{c \tilde{t}}{\lambda}, u=\frac{\tilde{u}}{c}, v=\frac{\tilde{v}}{c \delta}, \delta=\frac{a}{\lambda}, w=\frac{a \widetilde{w}}{c}, h=\frac{\tilde{h}}{a}, \\
& \left.l=\frac{\tilde{l}}{\lambda}, \phi=\frac{\tilde{\phi}}{a}, \sigma=\frac{\tilde{\sigma}}{a^{2}}, p=\frac{\tilde{p} a^{2}}{\mu c \lambda}, \operatorname{Re}=\frac{\rho c a \delta}{\mu}, Q=\frac{\widetilde{Q}}{\pi a^{2} c}, \quad\right\}
\end{aligned}
$$

where $\delta=a / \lambda$ is wave number, Re is the Reynolds number and $Q$ is volume flow rate.

Employing long wavelength and low Reynolds number approximations, the dimensionless equations obtained from Eqs. (2-5) reduce to

$$
\begin{aligned}
& \frac{\partial p}{\partial x}=\frac{1}{1-N}\left\{\frac{N}{r} \frac{\partial(r w)}{\partial r}+\frac{1}{r} \frac{\partial}{\partial r}\left(r \frac{\partial u}{\partial r}\right)\right\}, \\
& \frac{\partial p}{\partial r}=0 \\
& 2 w+\frac{\partial u}{\partial r}-\frac{2-N}{M^{2}} \frac{\partial}{\partial r}\left(\frac{1}{r} \frac{\partial(r w)}{\partial r}\right)=0 \\
& \frac{\partial u}{\partial x}+\frac{1}{r} \frac{\partial(r v)}{\partial r}=0
\end{aligned}
$$

where $N=k /(\mu+k)$ is the coupling number i.e., a measure of particle coupling with its surroundings $(0 \leq N \leq 1), \quad M=\sqrt{a^{2} k(2 \mu+k) / \gamma(\mu+k)}$, is the micro-polar parameter and $\alpha, \beta$ do not appear in the governing equation as the micro-rotation vector is solenoidal. In the limiting case, $k \rightarrow 0$, i.e., $N \rightarrow 0$, the governing equations for the micro-polar fluid reduce to the governing equations for Newtonian fluid.

$$
h(x, t)=1-\phi \cos ^{2} \pi(x-t) .
$$

The following are the boundary conditions imposed on the governing equations:

$$
\begin{aligned}
& \left.u(x, t)\right|_{r=h}=0,\left.\quad v(x, t)\right|_{r=h}=\frac{\partial h}{\partial t}, \\
& \left.v(x, t)\right|_{r=0}=0,\left.\quad \frac{\partial u(x, t)}{\partial r}\right|_{r=0}=0 . \\
& \left.w(x, t)\right|_{r=0}=0,\left.\quad w(x, t)\right|_{r=h}=0 .
\end{aligned}
$$

\subsection{Analysis}

Integration of Eq. (8) once with respect to $r$ yields

$$
\frac{\partial u}{\partial r}=(1-N) \frac{r}{2} \frac{\partial p}{\partial x}-N w+\frac{C_{1}}{r} .
$$

Further, integrating Eq. (10) twice with respect to $r$, and also using Eq. (15), the micro-polar vector is obtained as 


$$
\begin{aligned}
w=C_{2} I_{1}(M r) & +C_{3} K_{1}(M r) \\
& -\frac{1}{2-N}\left\{(1-N) \frac{r}{2} \frac{\partial p}{\partial x}+\frac{C_{1}}{r}\right\},
\end{aligned}
$$

where $C_{1}, C_{2}, C_{3}$ are arbitrary functions independent of $r$ and $I_{1}(M r), K_{1}(M r)$ are respectively the modified Bessel functions of the first and the second kind of the first order.

Then, applying fourth boundary condition of Eq. (13), and the boundary conditions (14), Eqs. (15) and (16) become

$$
\begin{aligned}
& \frac{\partial u}{\partial r}=\frac{1-N}{2-N} \frac{\partial p}{\partial x}\left\{r-\frac{N h}{2} \frac{I_{1}(M r)}{I_{1}(M h)}\right\}, \\
& w=\frac{1-N}{2(2-N)} \frac{\partial p}{\partial x}\left\{\frac{h I_{1}(M r)}{I_{1}(M h)}-r\right\}
\end{aligned}
$$

and further integrating Eq. (17) and applying the noslip condition of Eq. (13), the axial velocity is found as

$$
\begin{aligned}
u=\frac{1-N}{2(2-N)} \frac{\partial p}{\partial x} & \left\{r^{2}-h^{2}+\frac{N h}{M}\right. \\
& \left.\left(\frac{I_{0}(M h)-I_{0}(M r)}{I_{1}(M h)}\right)\right\},
\end{aligned}
$$

where $I_{0}(M r), I_{0}(M h)$ are the modified Bessel functions of the first kind and the zeroth order.

The radial velocity is derived from Eq. (11), by substituting $u$ from Eq. (19) and integrating it once with respect to $r$. The regularity condition, given in Eq. (13), determines the constant term and gives the radial velocity as

$$
\begin{aligned}
v=\frac{1-N}{2(2-N)}[ & \frac{\partial p}{\partial x} \frac{\partial h}{\partial x}\left\{r h-\frac{N}{M}\left\{\frac{r}{2} \frac{\partial}{\partial x}\left(\frac{h I_{0}(M h)}{I_{1}(M h)}\right)-\frac{I_{1}(M r)}{M} \frac{\partial}{\partial x}\left(\frac{h}{I_{1}(M h)}\right)\right\}\right\} \\
& \left.-\frac{\partial^{2} p}{\partial x^{2}}\left\{\frac{r^{3}}{4}-\frac{r h^{2}}{2}+\frac{N h}{M I_{1}(M h)}\left(\frac{r}{2} I_{0}(M h)-\frac{1}{M} I_{1}(M h)\right)\right\}\right] .
\end{aligned}
$$

In order to get pressure gradient, we apply the radial velocity of the wall, given in Eq. (13), on Eq. (20). This gives

$$
\begin{aligned}
h \frac{\partial h}{\partial t}=\frac{1-N}{2(2-N)} & {\left[\frac{\partial p}{\partial x} \frac{\partial h}{\partial x}\left\{h^{3}-\frac{N h}{M}\left(\frac{h}{2} \frac{\partial}{\partial x}\left(\frac{h I_{0}(M h)}{I_{1}(M h)}\right)-\frac{I_{1}(M h)}{M} \frac{\partial}{\partial x}\left(\frac{h}{I_{1}(M h)}\right)\right)\right\}\right.} \\
& \left.+\frac{\partial^{2} p}{\partial x^{2}}\left\{\frac{h^{4}}{4}+\frac{N h^{2}}{2 M^{2}}\left(2-\frac{M h I_{0}(M h)}{I_{1}(M h)}\right)\right\}\right],
\end{aligned}
$$


where $G(t)$ is a function of $t$ which is evaluated by a simple manipulation as which yields, on performing the integration, the following expression:

$$
G(t)=\frac{\frac{1-N}{8(2-N)}(p(l, t)-p(0, t))-\int_{0}^{l} \frac{\int_{0}^{x} h \frac{\partial h}{\partial t} d s}{h^{4}+\frac{4 N h^{2}}{M^{2}}\left(1-\frac{M h I_{0}(M h)}{2 I_{1}(M h)}\right)} d x}{\int_{0}^{l} \frac{1}{h^{4}+\frac{4 N h^{2}}{M^{2}}\left(1-\frac{M h I_{0}(M h)}{2 I_{1}(M h)}\right)} d x},
$$

where $\Delta p_{l}(t)=p(l, t)-p(0, t)$, is the pressure difference between the inlet and the outlet of the tube.

The volume flow rate is defined as

$$
Q(x, t)=\int_{0}^{h} 2 r u d r,
$$

$$
\begin{aligned}
Q(x, t)= & \frac{N-1}{4(2-N)} \frac{\partial p}{\partial x}\left\{h^{4}+\frac{4 N h^{2}}{M^{2}}\right. \\
& \left.\left(1-\frac{M h I_{0}(M h)}{2 I_{1}(M h)}\right)\right\} .
\end{aligned}
$$

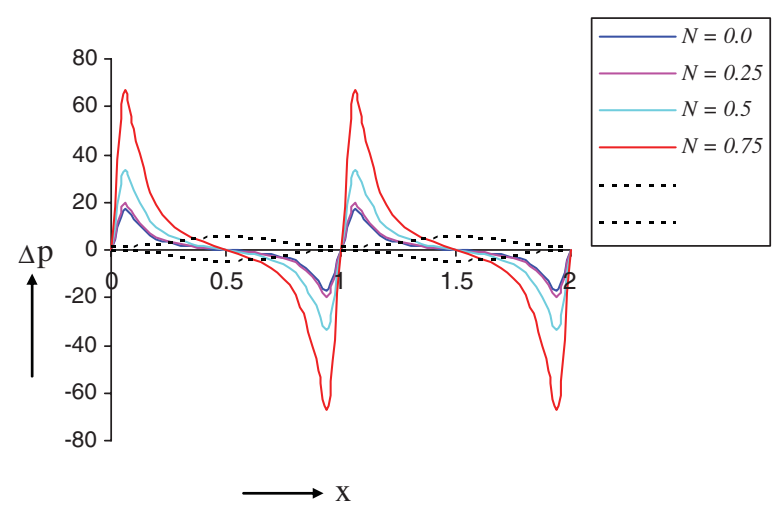

(a) $t=0.0,1.0$

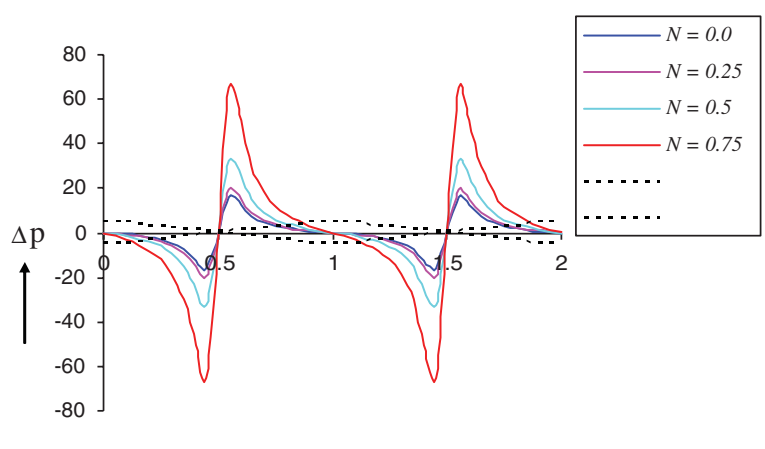

(c) $t=0.5$

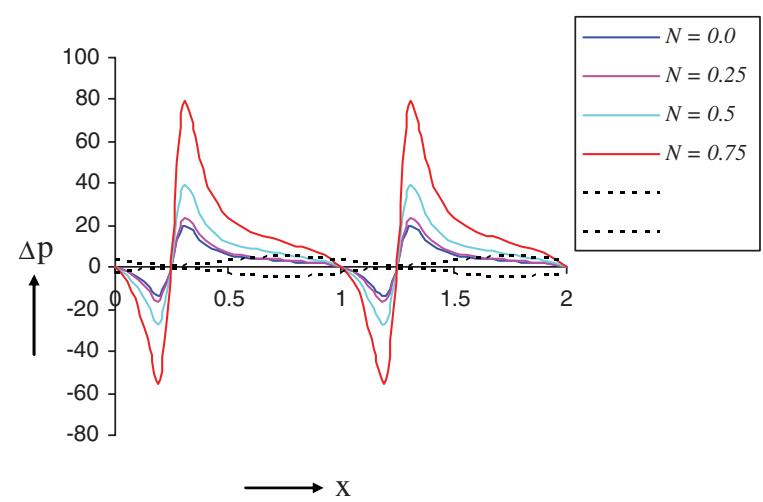

(b) $t=0.25$

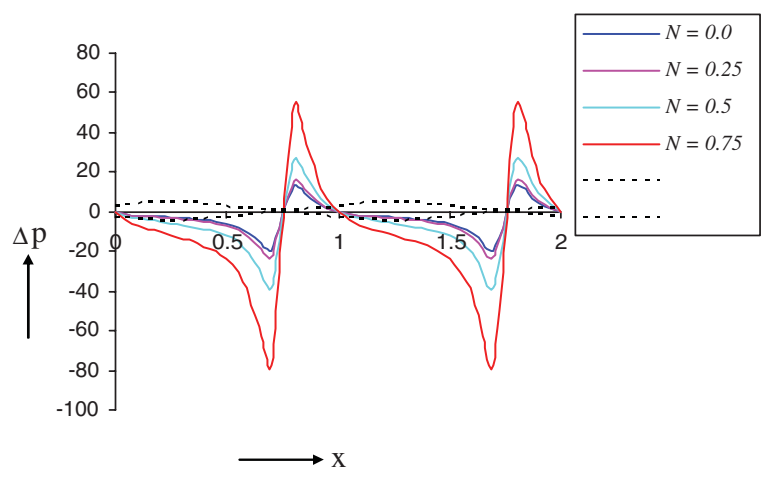

(d) $t=0.75$

Fig. 2. Pressure distribution along the axial distance of the finite length tube at five time instants based on Eq. (23). Dotted lines ( . . . . .) represent the position of wave, whereas continuous lines (-) represent the pressure distribution for $N=0.0-1.0, \phi=0.9, N=1.0$ and $l=2.0$. 
The time-averaged volume flow rate is obtained by averaging the volume flow rate for one time period. This gives

$$
\begin{aligned}
\bar{Q}= & \frac{N-1}{4(N-2)} \int_{0}^{1} \frac{\partial p}{\partial x}\left\{h^{4}+\frac{4 N h^{2}}{M^{2}}\right. \\
& \left.\left(1-\frac{M h I_{0}(M h)}{2 I_{1}(M h)}\right)\right\} d t .
\end{aligned}
$$

The time-averaged volume flow rate can be given in terms of the flow rate in the wave frame, and also in the laboratory frame, as

$$
\begin{aligned}
\bar{Q} & =q+1-\phi+\frac{3 \phi^{2}}{8} \\
& =Q-h^{2}+1-\phi+\frac{3 \phi^{2}}{8} .
\end{aligned}
$$

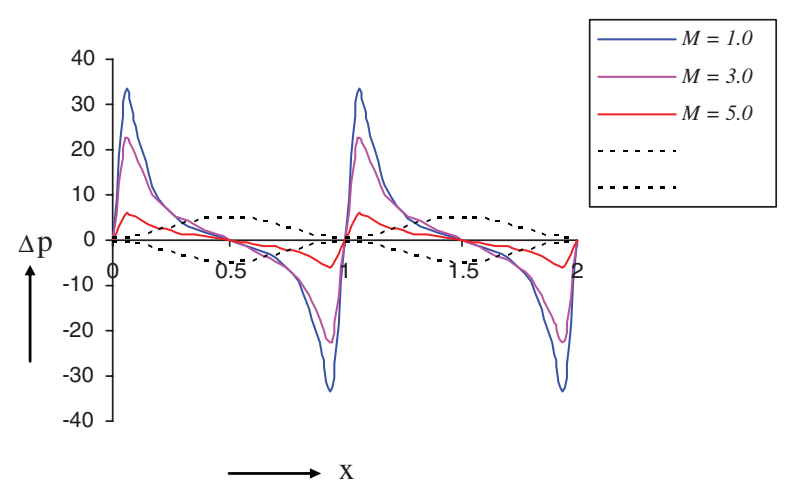

(a) $t=0.0,1.0$

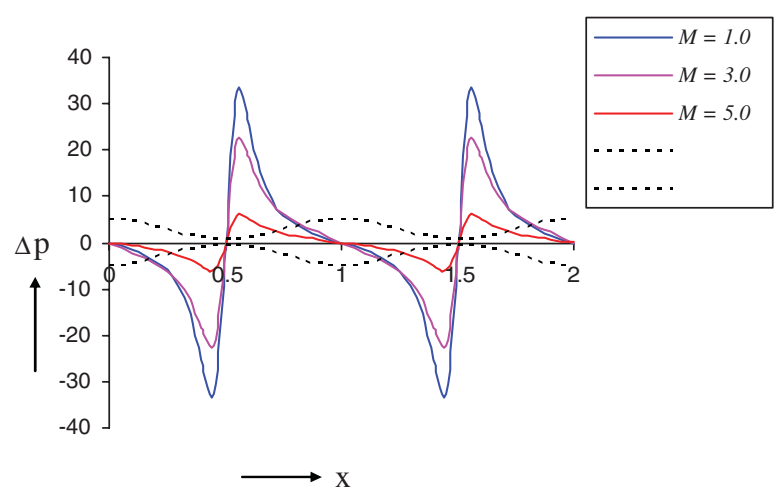

(c) $t=0.5$
This helps us express the pressure gradient in terms of the time-averaged volume flow rate. With some manipulations Eqs. (26) and (28) give

$$
\begin{aligned}
\frac{\partial p}{\partial x}= & \frac{4(2-N)}{N-1} \\
& \left\{\frac{\bar{Q}+h^{2}-1+\phi-\frac{3 \phi^{2}}{8}}{h^{4}+\frac{4 N h^{2}}{M^{2}}\left(1-\frac{M h I_{0}(M h)}{2 I_{1}(M h)}\right)}\right\},
\end{aligned}
$$

on integration, which yields pressure difference, in terms of the time-averaged volume flow rate, as

$$
\begin{aligned}
p(x)-p(0)= & \frac{4(2-N)}{N-1} \\
& \int_{0}^{x} \frac{\bar{Q}+h^{2}-1+\phi-\frac{3 \phi^{2}}{8}}{h^{4}+\frac{4 N h^{2}}{M^{2}}\left(1-\frac{M h I_{0}(M h)}{2 I_{1}(M h)}\right)} d s,
\end{aligned}
$$

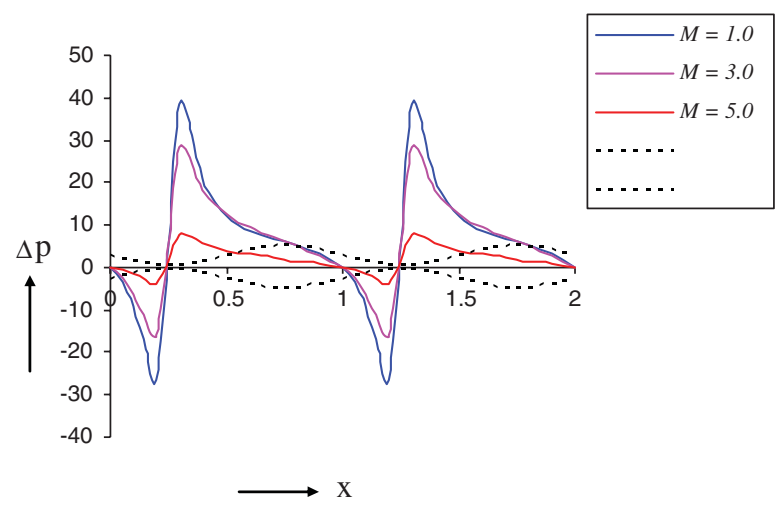

(b) $t=0.25$

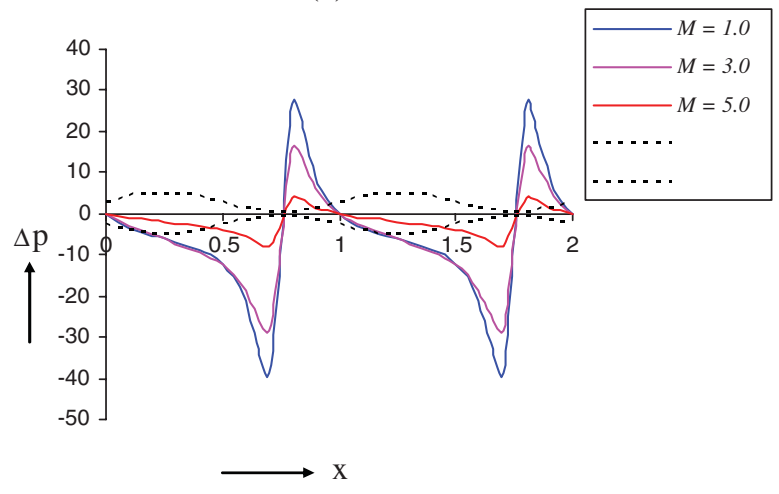

(d) $t=0.75$

Fig. 3. Pressure distribution along the axial distance of the finite length tube at five time instants based on Eq.(23). Dotted lines ( $\cdots \cdots$. . represent the position of wave, whereas continuous lines (-) represent the pressure distribution for $M=1.0-5.0, \phi=0.9, N=0.50$ and $l=2.0$. 
which gives for $x=l$

$$
\begin{aligned}
p(l)-p(0)= & \frac{4(2-N)}{N-1} \\
& \int_{0}^{l} \frac{\bar{Q}+h^{2}-1+\phi-\frac{3 \phi^{2}}{8}}{h^{4}+\frac{4 N h^{2}}{M^{2}}\left(1-\frac{M h I_{0}(M h)}{2 I_{1}(M h)}\right)} d x .
\end{aligned}
$$

Finally, the local wall shear stress is defined as

$$
\tau_{w}=\left.\frac{\partial u}{\partial r}\right|_{r=h}
$$

which, by virtue of Eq. (17), takes the form

$$
\tau_{w}=\frac{(1-N) h}{2} \frac{\partial p}{\partial x}
$$
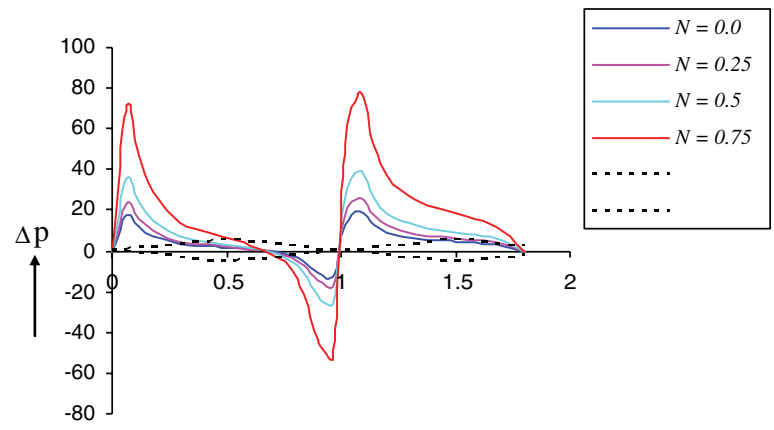

(a) $t=0.0,1.0$

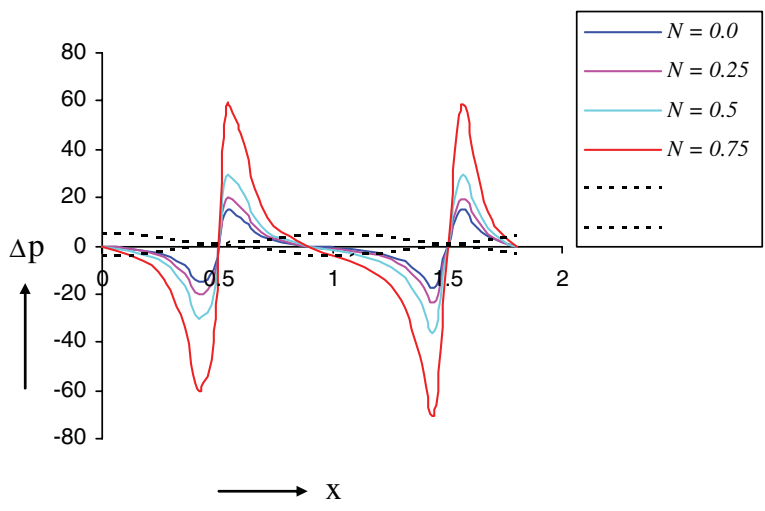

(c) $t=0.5$ and further reduces, in view Eq. (22), to

$$
\tau_{w}=4(2-N)\left\{\frac{G(t)+\int_{0}^{x} h \frac{\partial h}{\partial t} d s}{h^{3}+\frac{4 N h}{M^{2}}\left(1-\frac{M h I_{0}(M h)}{2 I_{1}(M h)}\right)}\right\} .
$$

\section{Mechanical efficiency}

Mechanical efficiency (cf. [21]) is derived for micropolar fluid as

$$
E=\frac{\bar{Q} \Delta p_{1}}{2 \phi\left[-(1+\phi) \Delta p_{1}+I_{11}+\phi I_{12}\right]},
$$

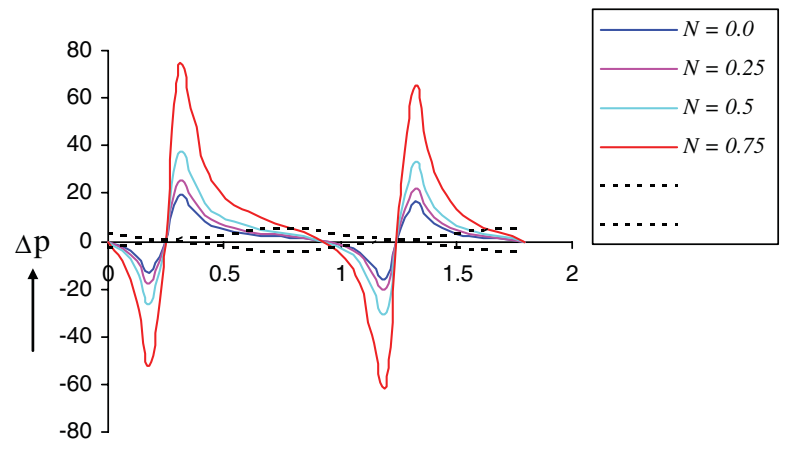

(b) $t=0.25$

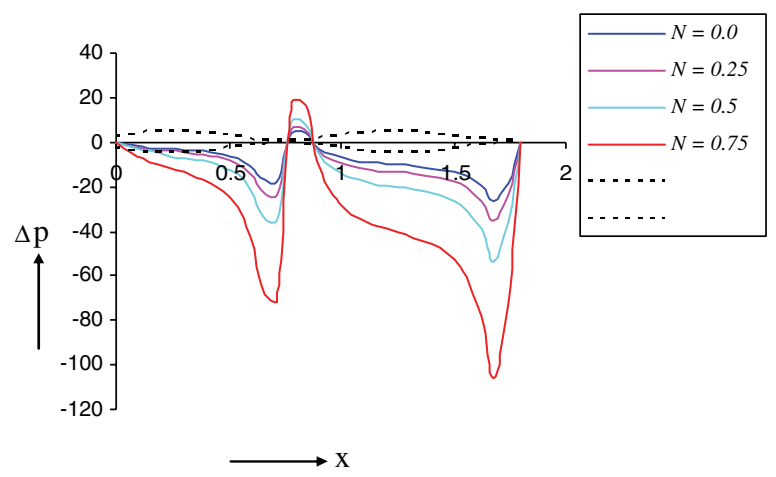

(d) $t=0.75$

Fig. 4. Pressure distribution along the axial distance of the finite length tube at five time instants based on Eq. (23). Dotted lines ( $\ldots \ldots)$ represent the position of wave, whereas continuous lines (-) represent the pressure distribution for $N=0.0-1.0, \phi=0.9, M=1.0$ and $l=1.8$. 
where $I_{11}=\int_{0}^{1} \frac{\partial p}{\partial x} \cos 2 \pi x d x, I_{12}=\int_{0}^{1} \frac{\partial p}{\partial x} \cos ^{4}(\pi x) d x$, and $\Delta p_{1}=p(1)-p(0)$ is the pressure difference across one wavelength and it is evaluated, by using Eq. (30), as

$$
\begin{aligned}
\Delta p_{1}= & \frac{4(2-N)}{N-1} \\
& \int_{0}^{1} \frac{\bar{Q}+h^{2}-1+\phi-\frac{3 \phi^{2}}{8}}{h^{4}+\frac{4 N h^{2}}{M^{2}}\left(1-\frac{M h I_{0}(M h)}{2 I_{1}(M h)}\right)} d x,
\end{aligned}
$$

and $\bar{Q}_{0}$, the maximum flow rate, is obtained by virtue of Eq. (34) and for $\Delta p_{1}=0$ as

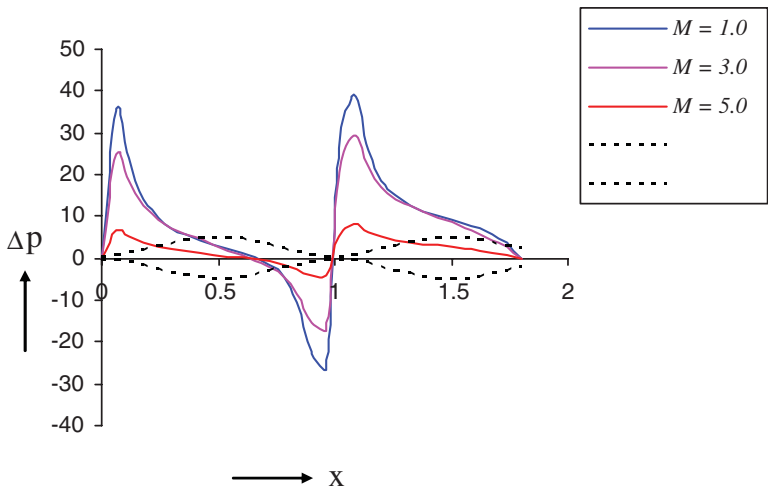

(a) $t=0.0,1.0$

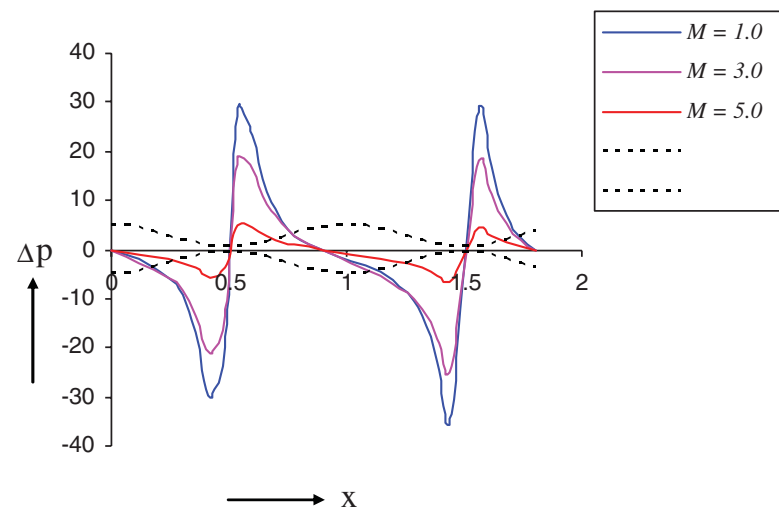

(c) $t=0.5$

$$
\bar{Q}_{0}=\left[1-\phi+\frac{3 \phi^{2}}{8}-\frac{\int_{0}^{1} \frac{1}{h^{2}+\frac{4 N}{M^{2}}\left(1-\frac{M h I_{0}(M h)}{2 I_{1}(M h)}\right)} d x}{\int_{0}^{1} \frac{1}{h^{4}+\frac{4 N h^{2}}{M^{2}}\left(1-\frac{M h I_{0}(M h)}{2 I_{1}(M h)}\right)} d x}\right] .
$$

\section{Reflux limit}

Reflux is an important phenomenon of peristaltic movement, which refers to the presence of fluid particles that move, on the average, in a direction opposite to the net flow in the close vicinity of the wall (cf. [21])

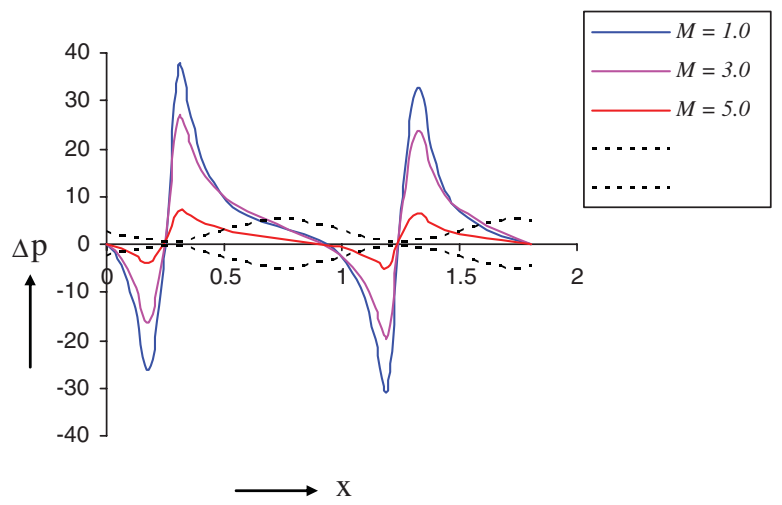

(b) $t=0.25$

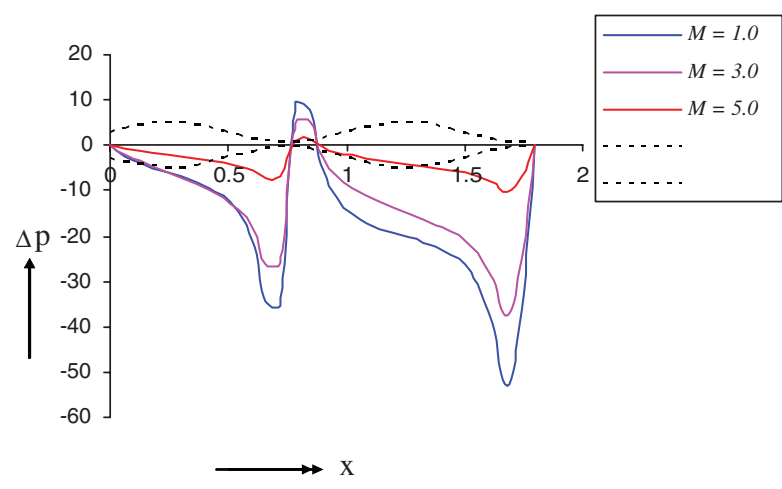

(d) $t=0.75$

Fig. 5. Pressure distribution along the axial distance of the finite length tube at five time instants based on Eq. (23). Dotted lines ( $\cdots \cdots$...) represent the position of wave, whereas continuous lines (-) represent the pressure distribution for $M=1.0-5.0, \phi=0.9, N=0.50$ and $l=1.8$. 
For the axisymmetric case, the dimensional form of the stream function in the wave frame is defined as

$$
d \tilde{\psi}=2 \pi \tilde{R}(\tilde{U} d \tilde{R}-\tilde{V} d \tilde{X})
$$

$$
\begin{array}{r}
\tilde{X}=\tilde{x}-c \tilde{t}, \quad \tilde{R}=\tilde{r}, \quad \tilde{U}=\tilde{u}-c, \quad \tilde{V}=\tilde{v}, \\
\tilde{q}=\tilde{Q}-c \tilde{h}^{2}, \quad \tilde{\Psi}=\tilde{\psi}-\tilde{r}^{2},
\end{array}
$$

where the left side of the parameters is in the wave frame while the right side of the parameters are in the laboratory frame, we obtain stream function as

$$
\psi=-\left[\frac{\left(\bar{Q}+h^{2}-1+\phi-\frac{3 \phi^{2}}{8}\right)\left\{r^{4}-2 r^{2} h^{2}+\frac{2 N h}{M^{2} I_{1}(M h)}\left(M I_{0}(M h) r^{2}-2 r I_{1}(M r)\right)\right\}}{h^{4}+\frac{4 N h^{2}}{M^{2}}\left(1-\frac{M h I_{0}(M h)}{2 I_{1}(M h)}\right)}+r^{2}\right]
$$

where $\tilde{\psi}, \tilde{X}, \tilde{R}, \tilde{U}$ and $\tilde{V}$ are stream function, axial coordinates, velocities components respectively.

Using the following transformations between the wave and the laboratory frames, defined as

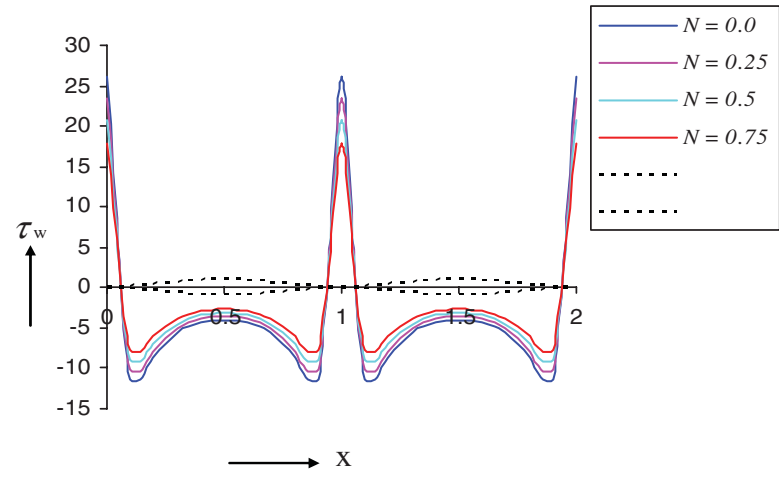

(a) $t=0.0,1.0$

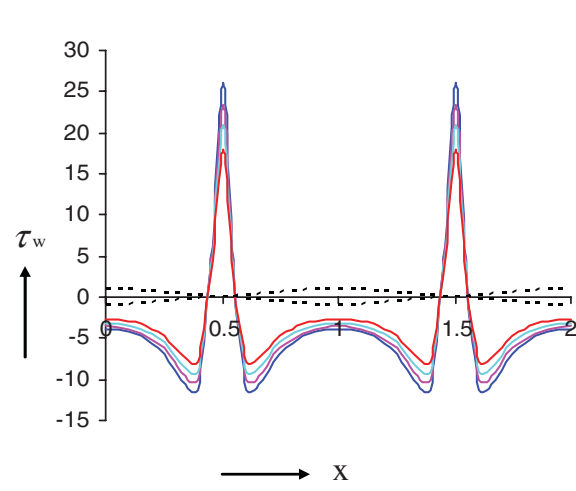

(c) $t=0.5$

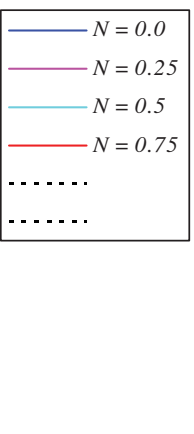

Stream function at the wall, $\psi_{w}$, is solved from Eq. (38) by substituting $r=h$. A simplification yields

$$
\left.\psi\right|_{r=h}=\psi_{w}=\bar{Q}-1+\phi-\frac{3 \phi^{2}}{8} .
$$

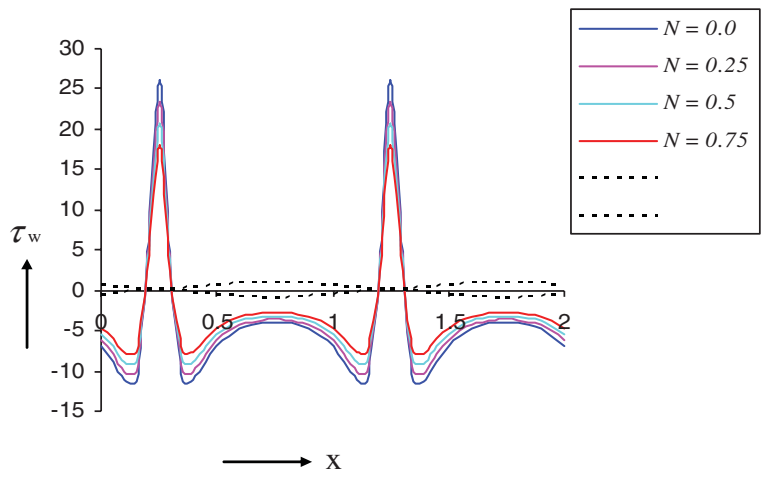

(b) $t=0.25$

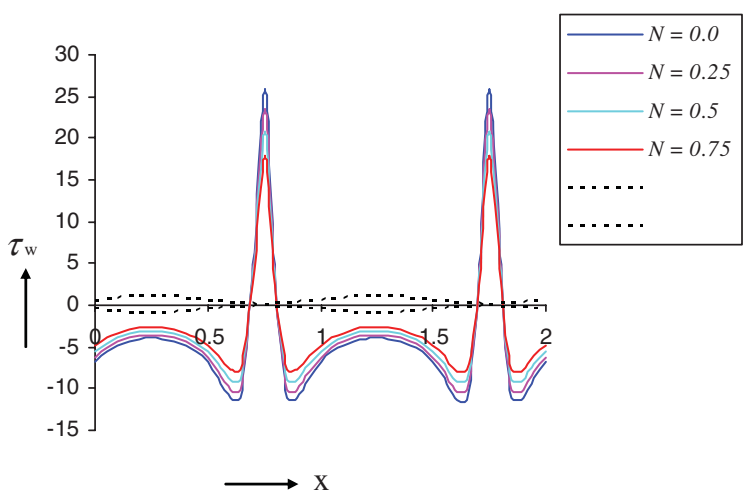

(d) $t=0.75$

Fig. 6. Local wall shear stress vs. axial distance along the finite length tube at five time instants based on Eq. (32). Dotted lines ( . . . .) represent the position of wave, whereas continuous lines (-) represent the local wall shear stress for $N=0.0-1.0, \phi=0.9, M=1.0$ and $l=2.0$. 
Reflux flow rate, $Q_{\psi}(x)$, associated with a particle at the position $x$, is given by

$$
Q_{\psi}(x)=\psi+r^{2}(\psi, x),
$$

which, on averaging over one cycle, gives

$$
\bar{Q}_{\psi}=\psi+\int_{0}^{1} r^{2}(\psi, x) d x
$$

Moreover, in order to evaluate the reflux limit, $\bar{Q}_{\psi}$ is expanded in a power series, in terms of a small parameter $\varepsilon$ about the wall, where $\varepsilon\left(=\psi-\psi_{w}\right)$ is subjected to the reflux condition

$$
\frac{\bar{Q}_{\psi}}{\bar{Q}}>1 \text { as } \varepsilon \rightarrow 0
$$

The coefficient of the first two terms in the expansion of $r$ is obtained only for small values of $M$. Substituting the expansion $r^{2}(\psi, x)=h^{2}+a_{1} \varepsilon+a_{2} \varepsilon^{2}+\cdots$ into Eq. (38), and using Eq. (39), we get

$$
\begin{gathered}
a_{1}=-1, \\
a_{2}=-\left[\frac{\left(1-\frac{1}{4} \frac{M N h}{I_{1}(M h)}\right)\left(\bar{Q}+h^{2}-1+\phi-\frac{3 \phi^{2}}{8}\right)}{h^{4}+\frac{4 N h^{2}}{M^{2}}\left(1-\frac{M h I_{0}(M h)}{2 I_{1}(M h)}\right)}\right] .
\end{gathered}
$$

Then integrating Eq. (40) with respect to $x$, and using Eqs. (41-44), we obtain the reflux limit (i.e. the occurrence of reflux) as

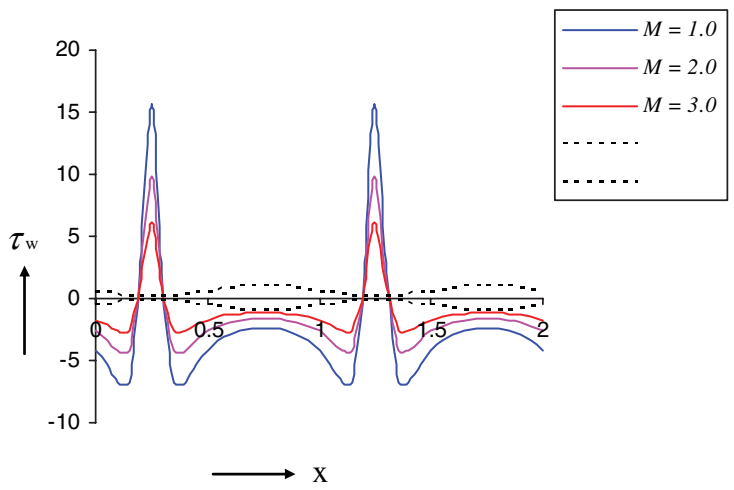

(b) $t=0.25$

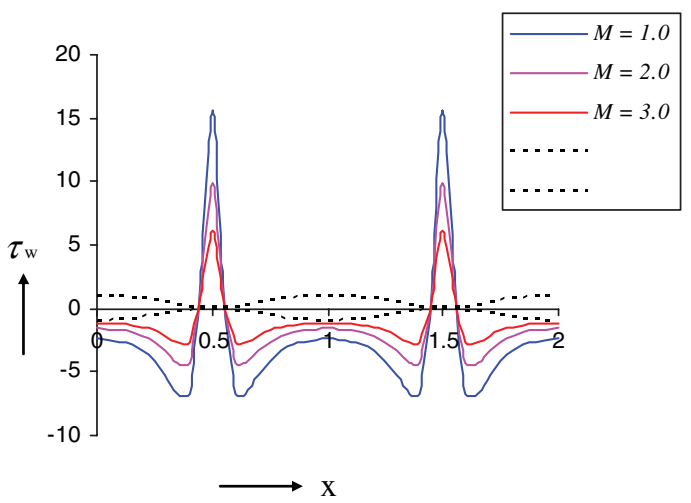

(c) $t=0.5$

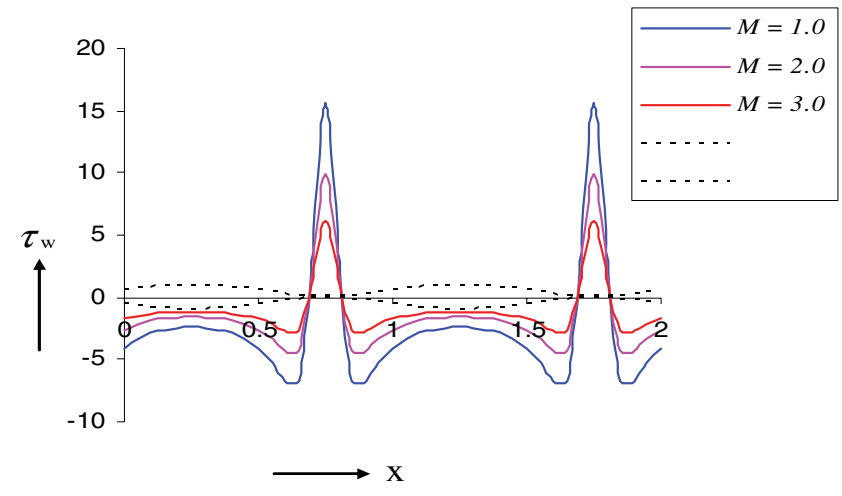

(d) $t=0.75$

Fig. 7. Pressure distribution by local wall shear stress at five time instants based on Eq. (32). Dotted lines ( $\cdots \cdots)$ represent the position of wave, whereas continuous lines (-) represent the local wall shear stress for $M=1.0-5.0, \phi=0.9, N=0.50$ and $l=2.0$. 


$$
\bar{Q}<1-\phi+\frac{3 \phi^{2}}{8}-\frac{\int_{0}^{1} \frac{\left(1-\frac{1}{4} \frac{M N h}{I_{1}(M h)}\right)}{h^{2}+\frac{4 N}{M^{2}}\left(1-\frac{M h I_{0}(M h)}{2 I_{1}(M h)}\right)} d x}{\int_{0}^{1} \frac{\left(1-\frac{1}{4} \frac{M N h}{I_{1}(M h)}\right)}{h^{4}+\frac{4 N h^{2}}{M^{2}}\left(1-\frac{M h I_{0}(M h)}{2 I_{1}(M h)}\right)} d x}(45)
$$

\section{Numerical results and discussion}

We apply the model to the flow through oesophagus; and therefore, we consider two boluses of a micro-polar fluid through an oesophagus. The case, we consider, is that of free pumping, i.e., the case when the pressure across the two ends (viz., the upper and lower sphincters) of the oesophagus is zero.

A keen examination of the effects of coupling number and micro-polar parameter, which together determine micro-polar character of the fluid, on the flow pattern, is carried out by computer simulation. We wrote programs in $\mathrm{C}$ and studied the graphs drawn based on the computational results.

First of all, we perform temporal examination of the pressure distribution along the length at various instants, i.e., for discrete values of $t=0.0-1.0$ (cf. Fig. 2). At $t=0$ (cf. Fig. 2a), it is observed that the pressure tends to rise sharply at the upper sphincter, reaches some peak, then falls at a lower rate to zero at the middle of the bolus, comes down further to a lower trench and finally rises sharply to meet the leading end of the bolus. The same distribution is repeated for the next bolus. The pressure at the lower sphincter is zero. This divulges the fact that the bolus is under complete control of the oesophagus. This is the beauty of this sort of pumping which is more for safe and controlled transportation than merely trying to pump with minimum effort for maximum load. After one fourth of the periodic cycle (cf. Fig. 2b), the bolus has moved ahead and a trailing bolus is on the way to entry, which is permitted by the upper sphincter by lowering the pressure which is once again zero at the head of it and rises very sharply so that the leading bolus does not suffer from any retrograde motion; whereas at the lower sphincter, pressure declines to zero in order to pave way for the outgoing bolus. The graphs for higher values of $t$ represent a systematic progress of the boluses in the oesophagus. Eventually, at the time $t=1$, which represents the completion of one period, the pressure distribution resembles that at $t=0$ : this indicates that a new cycle is ready to set out (cf. Fig. 2a).

Such a distribution was reported by Dodds [12], Ren et al. [13] and Brasseur and Dodds [15], who measured intrabolus pressures, computed using radiographic data of bolus shapes as input and compared with manometric measurements.

It is further observed that the pressure distribution along the oesophageal length for micro-polar fluid shows qualitative similarity with Newtonian fluid (cf. Fig. 2) which is a special case, i.e., $N \rightarrow 0$.

It also reveals that as the coupling effect parameter $N$ increases, pressure gradient as well as pressure along the length of the oesophagus enhances which may be physically interpreted as that inner-rotation of the fluid particles increases pressure; and finally when $N \rightarrow 0$, i.e., the fluid turns Newtonian, the pressure is minimum. This may lead to the conclusion that the oesophagus has to make additional efforts to swallow a micro-polar fluid. A similar observation is made for all values of $t$ ranging from $0 \rightarrow 1$, i.e., throughout one time period. Temporal effects are similar to those observed for Newtonian fluids, power-law fluids, visco-elastic fluids, visco-plastic fluids and magnetohydrodynamic fluids (cf. [16-20]). Figures, together with captions, provide the details (cf. Fig. 2).

We further carry out investigation into the role of the other micro-polar parameter $M$. It is observed that the pressure along the entire length of the oesophagus diminishes as $M$ increases. Hence, this parameter has an opposite influence vis-à-vis coupling number (cf. Fig. 3). Since no value of $M$ can lead to Newtonian nature, no comparison can be made with Newtonian fluids. In fact, the micro-polar fluid has a complex characteristic that is built up by the combined effects of these two parameters. This may be noticed that once $N=0, M$ seizes to affect the flow (cf. Eq. (23)).

Simultaneously, we consider the propagation of a non-integral number of waves in the train, which is an inherent feature of finite length vessels. Some significant differences are observed between this case and the other where there are an integral number of waves in the train. As a special case, we take $l=1.8$.

It is observed that unlike the case of an integral number of waves, the pressure distributions are different for the boluses enclosed within the whole and the fractional waves. Whatever be the temporal value, the pressure for the whole wave attains zero thrice: at the two ends and once midway depending upon the value of $t$ for the whole bolus while it reaches zero only twice at the two ends in the case of fractional bolus. But one significant difference between the two cases is that the peaks of pressure for the two different types of boluses are identical in the integral case while the peaks are 


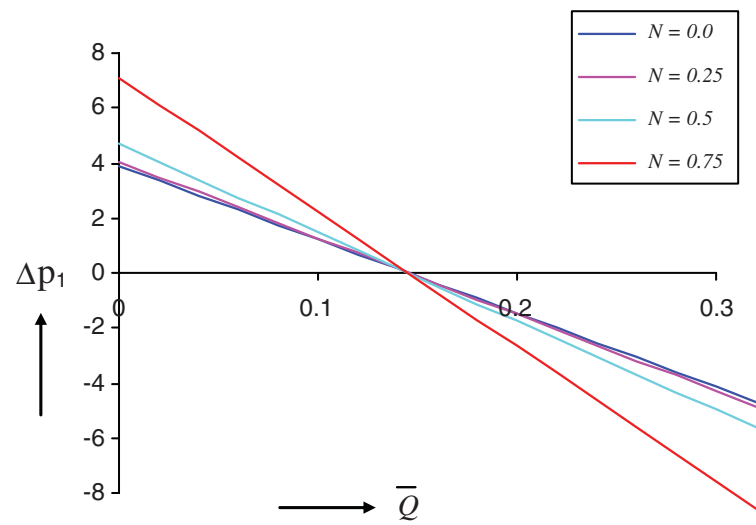

Fig. 8. Pressure difference across one wavelength vs. average flow rate based on Eq. (34) for $N=0.0-1.0, \phi=0.4$ and $M=1.0$.

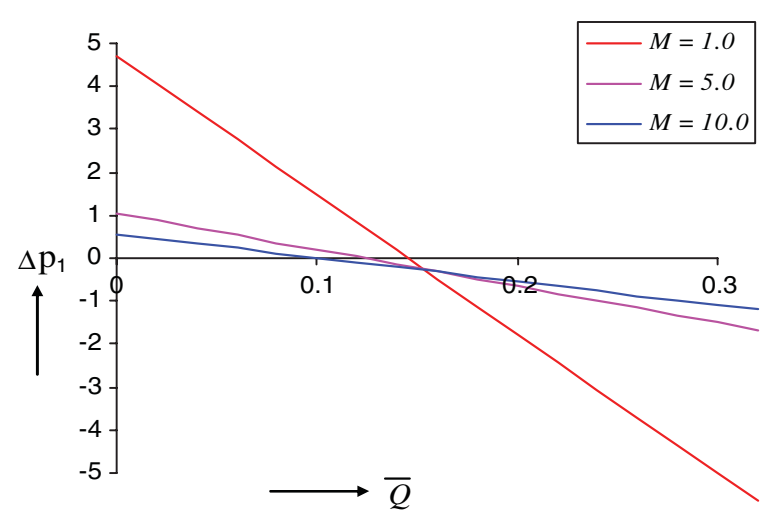

Fig. 9. Pressure difference across one wavelength vs. average flow rate based on Eq. (34) for $M=1.0-5.0, \phi=0.4$ and $N=0.50$.

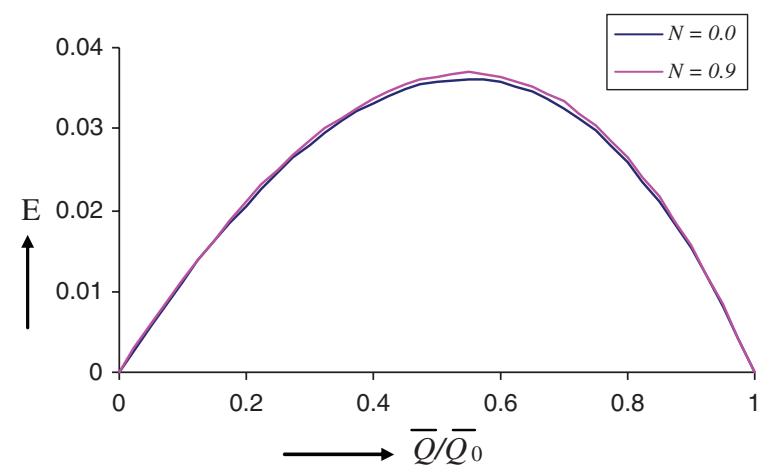

Fig. 10. Mechanical efficiency vs. ratio of average flow rate to maximum average flow rate, based on Eq. (33), curves represent efficiency of peristaltic pump for $N=0.0,0.9, \phi=0.4$ and $M=1.0$.

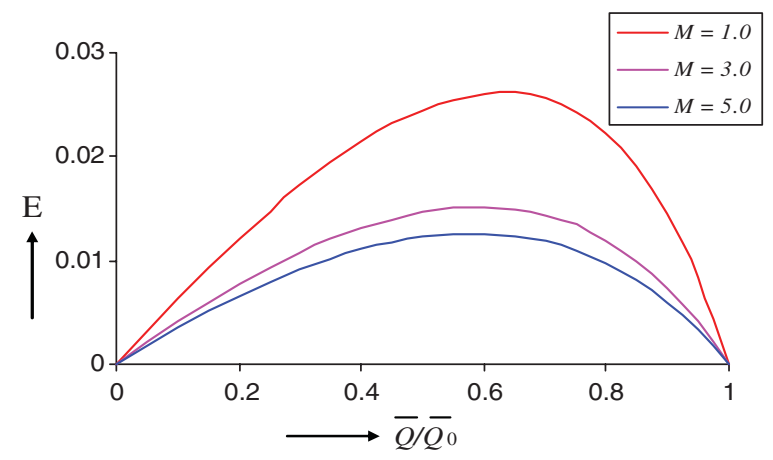

Fig. 11. Mechanical efficiency vs. ratio of average flow rate to maximum average flow rate, based on Eq. (33), curves represent efficiency of peristaltic pump for $M=1.0,3.0,5.0, \phi=0.4$ and $N=0.50$.

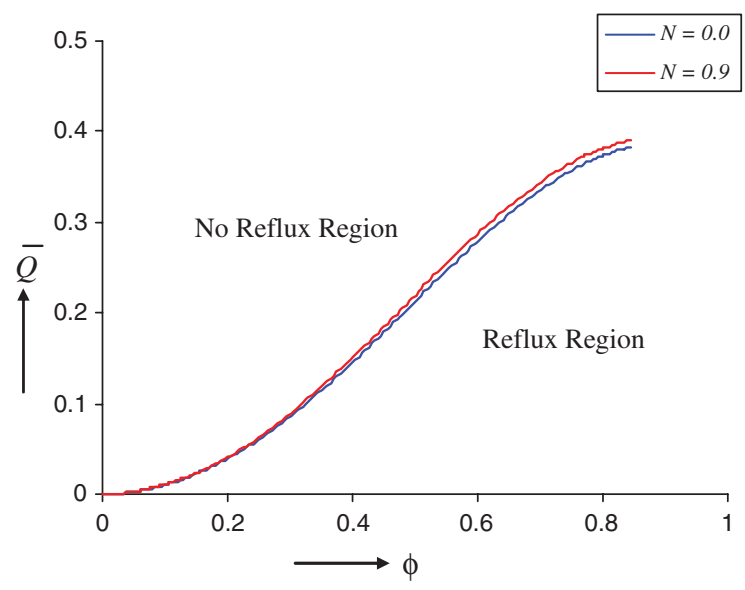

Fig. 12. Average flow rate vs. amplitude, based on Eq. (45). Curves represent reflux limit for $N=0.0,0.9, \phi=0.4$ and $M=0.1$.

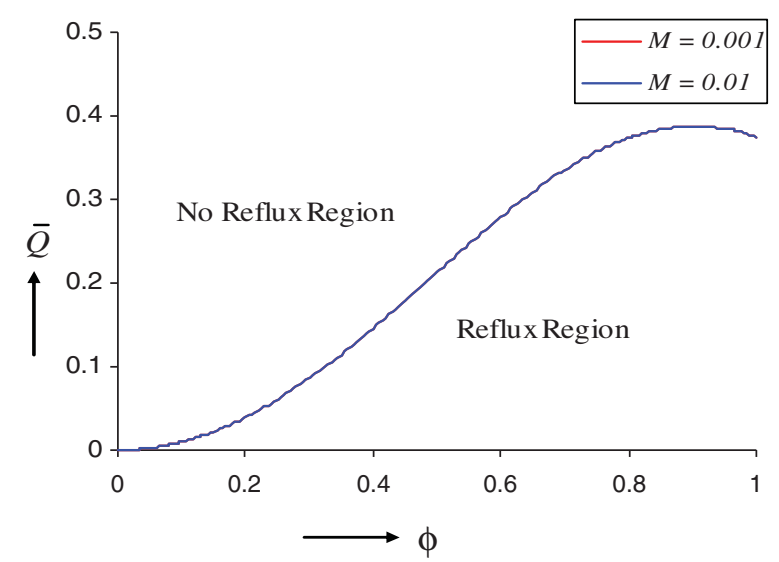

Fig. 13. Average flow rate vs. amplitude, based on Eq. (45). Curves represent reflux limit for $M=0.001,0.01, \phi=0.4$ and $N=0.5$. 
different in the non-integral case (cf. Fig. 4). Pressure distribution changes with $t$. It is further observed that the closer to symmetry is the distribution of boluses in the vessel, the lesser is the difference between the pressure peaks (Fig. 4c). The effects of the coupling number $N$ and the micro-polar parameter $M$ do not reveal any difference in the two cases (cf. Figs. 2-5).

Figures 6 and 7 depict the temporal effects of coupling number $N$ and micro-polar parameter $M$ on local wall shear stress along the length of tube for various values of $t=0.0-1.0$. It is observed that the local wall shear stress increases with coupling number $N$, whereas it decreases with micro-polar parameter $M$.

A graph, plotted for pressure and averaged flow rate that exhibits a linear relation between them, for varying coupling number $N$ and given $M$, indicates that pressure increases with coupling number $N$. For a given coupling number $N$, it is similar to that for Newtonian fluids (cf. Fig. 8). Observation is that the pressure decreases with increasing $M$ and for given $N$ (cf. Fig. 9).

Mechanical efficiency is an important characteristic of a pump. Graphs plotted for the mechanical efficiency reveal that it increases with the coupling number $N$ (cf. Fig. 10) for given $M$ and decreases with the micropolar parameter $M$ for given $N$ (cf. Fig. 11). Captions of the figures give details of the other parameters. The fluid turns Newtonian as $N \rightarrow 0$. This is noteworthy that while examining pumping efficiency, we have fixed the flow rate so that higher efficiency is an indication that the pump has to be more efficient to transfer the same amount of micro-polar fluids. In other words, efficiency is less, in case a micro-polar fluid is pumped.

Reflux relates to the retrograde motion of the fluid particles close to the inner surface of the wall of the vessel in which the flow is considered. It has been discussed here only for small values of micro-polar parameter. The graphs based on Eq. (46) for reflux limit discloses that the reflux region for micro-polar fluid increases with the coupling number $N$ (cf. Fig. 12). Physically it can be interpreted as that the flow becomes more prone to reflux when inner rotation of fluid particles increases.

Since $M$ is considered very small, no significant difference is observed (cf. Fig. 13); and even if it is measured very carefully, it won't give us accurate values. For this reason, discussion in this regard is dropped.

Another important phenomenon called trapping refers to closed circulating streamlines that exist at very

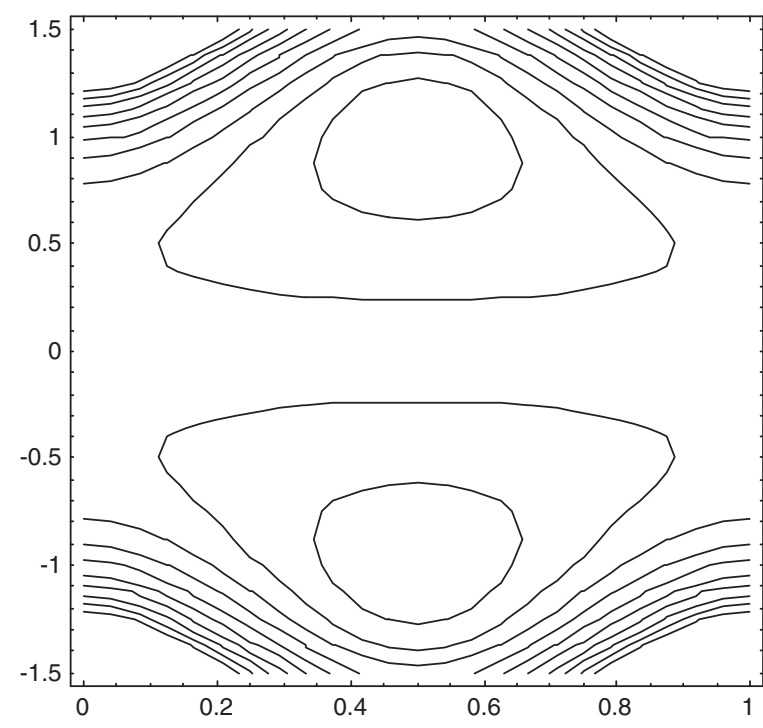

Fig. 14. Streamlines in the wave frame when $\bar{Q}=0.9, \phi=0.6$, $M=0.001, N=0.25$.

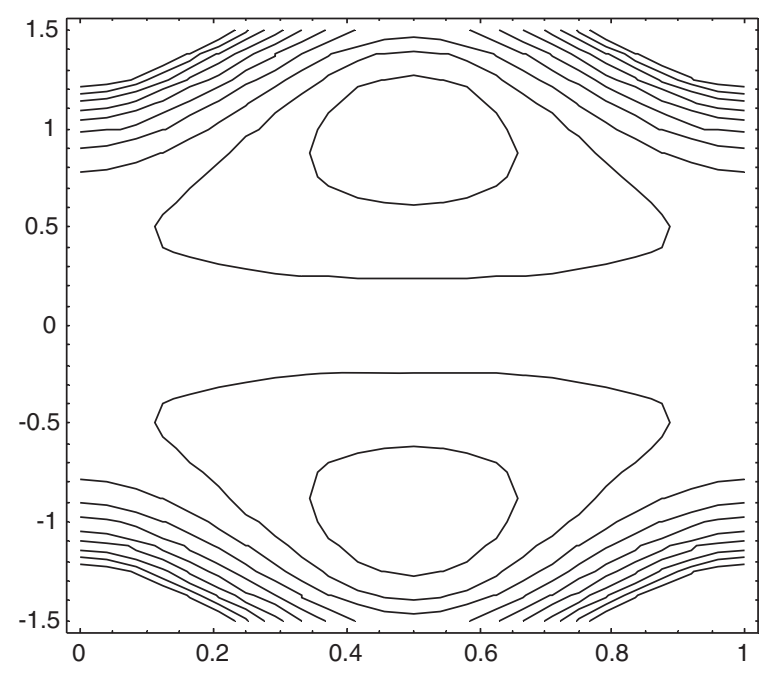

Fig. 15. Streamlines in the wave frame when $\bar{Q}=0.9, \phi=0.6$, $M=0.01, N=0.25$.

high flow rates and when occlusions are very large (cf. [21]). The trapped region moves with the wavevelocity. It has been studied for varying micro-polar parameter $M$ and coupling number $N$. We plot streamlines based on Eq. (38) for this case. Illustrating Figs (14-17) reveal that when $M$ is increased by keeping other parameters unchanged, the size of the trapped region decreases slightly. Unlike this, the size of the trapped region increases when $N$ enhances with other 


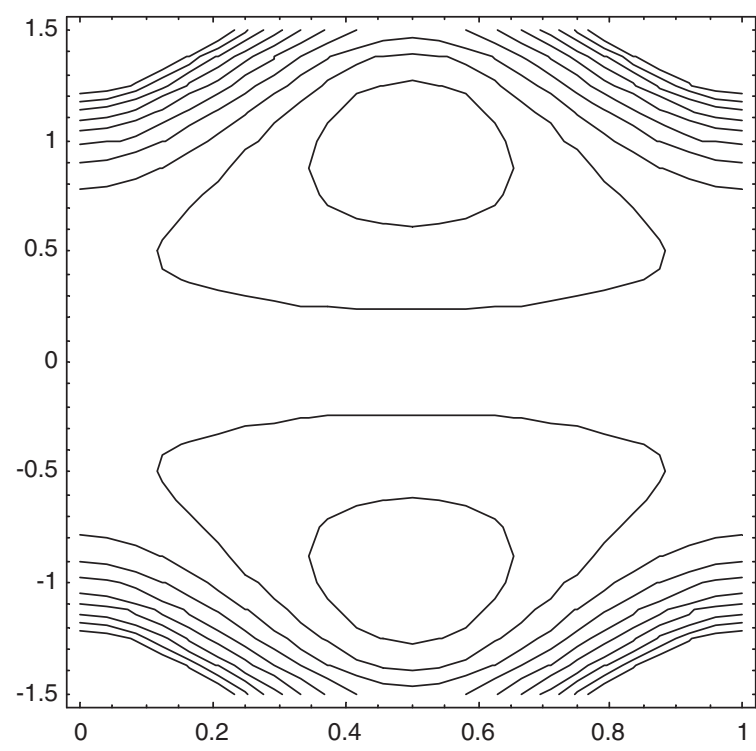

Fig. 16. Streamlines in the wave frame when $\bar{Q}=0.9, \phi=0.6$, $M=0.1, N=0.25$.

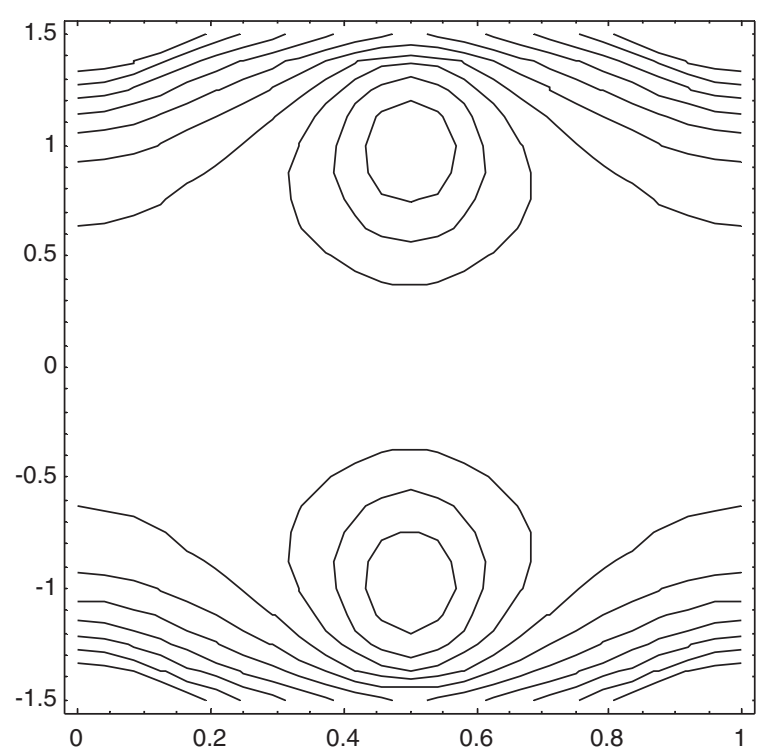

Fig. 17. Streamlines in the wave frame when $\bar{Q}=0.9, \phi=0.6$, $M=1.0, N=0.25$.

parameters remaining unaltered [cf. Figs. 17-19]. Ali and Hayat [10] reported similar observations for $N$ and $M$ for asymmetric channels. However, Mekheimer and Elmaboud [11] and Hayat et al. [6] share a different opinion regarding the effects of $M$ on trapping. They

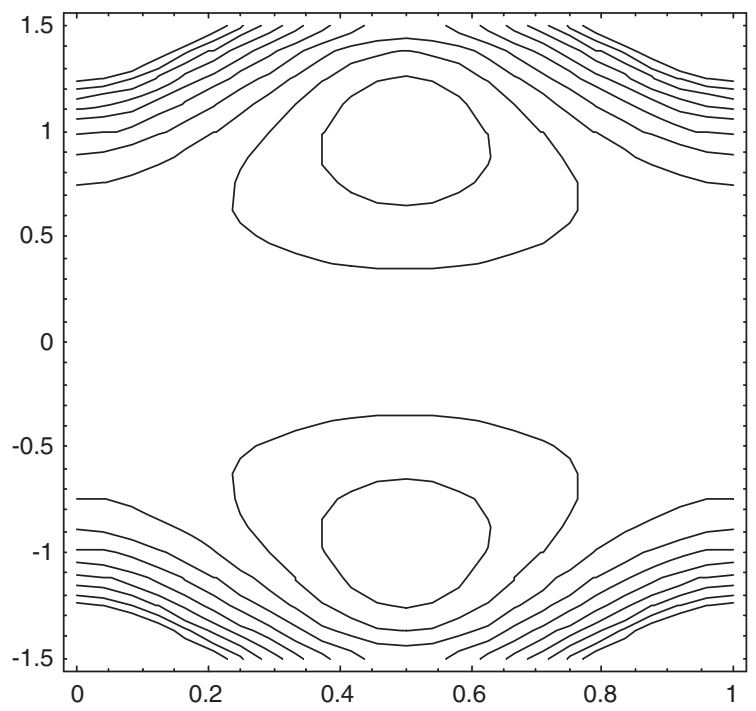

Fig. 18. Streamlines in the wave frame when $\bar{Q}=0.9, \phi=0.6$ $M=1.0, N=0.5$.

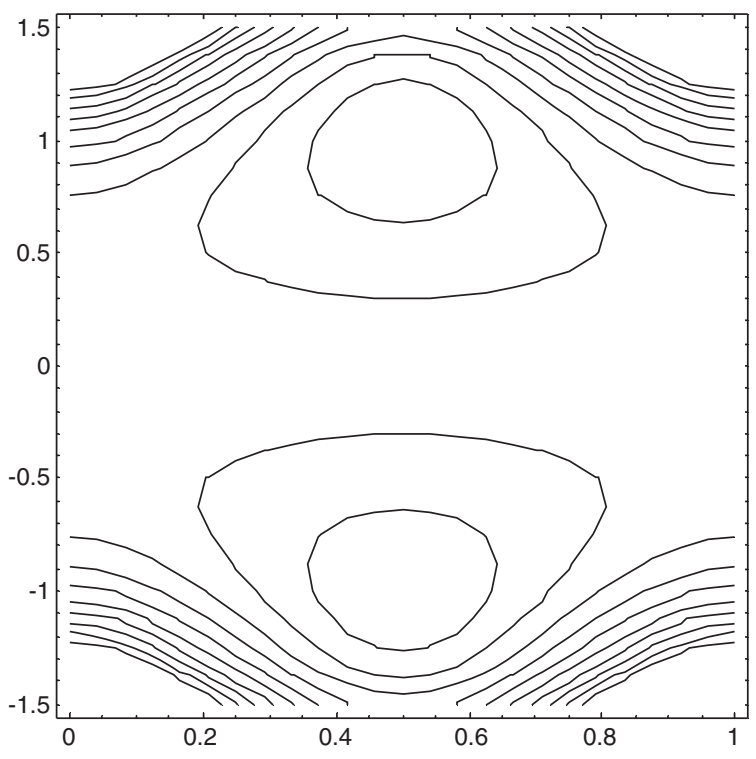

Fig. 19. Streamlines in the wave frame when $\bar{Q}=0.9, \phi=0.6$, $M=1.0, N=0.75$.

try to examine this for arbitrary flow rates by not caring whether the flow rates and occlusions are very high or not which are essential for trapping. Probably for this reason, they are inconclusive about the influence of $M$ on trapping. 


\section{Conclusions}

Newtonian and micro-polar fluids have qualitatively similar pressure distributions; but differences in magnitudes are very much significant. Divulgation is that coupling number increases pressure along the entire length of the oesophagus, while the other parameter decreases it. The latter one favours the flow, whereas the former one hinders it. The combined effect will be no doubt a complicated one. Had the exact experimental values of both parameters been available in the literature, we would have evaluated.

A pump with micro-polar fluid is also affected by the two parameters. Coupling number increases the efficiency; while the other micro-polar parameter decreases it. It is further concluded that the pumping efficiency decreases for micro-polar fluids.

Reflux region is found to increase with the coupling number. This may be inferred that a micro-polar fluid is more prone to flow reversal, i.e., reflux.

The peaks of pressure are identical in the integral case while the peaks have different altitudes in the nonintegral case. The effects of the coupling number and the micro-polar parameter are identical in both cases integral and non-integral number of waves in train.

Trapping is favoured by the coupling number but hindered by the micro-polar parameter.

\section{References}

[1] A.C. Eringen, Theory of micropolar fluids, J Math Mech 16 (1966), 1-16.

[2] G. Devi and R. Devanathan, Peristaltic motion of micropolar fluid, Proc Indian Acad Sci 81(A) (1975), 149-163.

[3] D. Philip and P. Chandra, Peristaltic transport of a simple micro fluid, Proc Nat Acad Sci India 65(A) (1995), 63-74.

[4] R. Bhargava, S. Sharma, H.S. Takhar, T.A. Bég, O.A. Bég and T.K. Hung, Peristaltic pumping of micropolar fluid in porous channel - model for stenosed arteries, J Biomech 39 (2006), S649

[5] D. Srinivasacharya, M. Mishra and A.R. Rao, Peristaltic transport of a micropolar fluid in a tube, Acta Mech 161 (2003), 165-178.
[6] T. Hayat, N. Ali and Z. Abbas, Peristaltic flow of a micropolar fluid in channel with different wave forms, Phys Lett A $\mathbf{3 7 0}$ (2007), 331-344.

[7] P. Muthu, B.V.K. Rathish and P. Chandra, On the influence of wall properties in the peristaltic motion of micropolar fluid, ANZIAM Journal 45 (2003), 245-260.

[8] P. Muthu, B.V.K. Rathish and P. Chandra, Peristaltic motion of micropolar fluid in circular cylindrical tubes: Effect of wall properties, Appl Math Model 32 (2008), 2019-2033.

[9] T. Hayat and N. Ali, Effects of an endoscope on peristaltic flow of a micropolar fluid, Math Comput Model 48 (2008), 721-733.

[10] N. Ali and T. Hayat, Peristaltic flow of a micropolar fluid in an asymmetric channel, Comput Math Appl 55 (2008), 589608.

[11] Kh. S. Mekheimer and Y. Abd Elmaboud, The influence of a micropolar fluid on peristaltic transport in an annulus: Application of the clot model, Applied Bionics and Biomechanics 5 (2008), 13-23.

[12] W.J. Dodds, Radiology of oesophagus, in: Alimentary Tract Radiology, 3rd edn, A.R. Margulis and H.J. Burhenne, eds, 1983, pp. 1529-1603.

[13] J. Ren, B.T. Massey, W.J. Dodds, M.K. Kern, J.G. Brasseur, R. Shaker, S.S. Harrington, W.J. Hogan and R.C. Arndorfer, Determinants of intrabolus pressure during esophageal peristaltic bolus transport, Am J Physiol 264 (1993), G407-G413.

[14] M. Li and J.G. Brasseur, Non-steady peristaltic transport in finite length tubes, J Fluid Mech 248 (1993), 129-151.

[15] J.G. Brasseur and W.J. Dodds, Interpretation of intraluminal manometric measurements in terms of swallowing mechanics, Dysphagia 6 (1991), 100-119.

[16] J.C. Misra and S.K. Pandey, A mathematical model for oesophageal swallowing of a food bolus, Math Comput Model 33 (2001), 997-1009.

[17] S.K. Pandey and D. Tripathi, Unsteady model of transportation of a Jeffrey-fluid by peristalsis through oesophagus, Int J Biomathematics 3 (2010), 453-472.

[18] S.K. Pandey and D. Tripathi, Peristaltic transport of a casson fluid in a finite channel: Application to flows of concentrated fluids in oesophagus, Int J Biomathematics 3 (2010), 473-491.

[19] S.K. Pandey and D. Tripathi, Influence of magnetic field on peristaltic flow of viscous fluid through a finite length cylindrical tube, Applied Bionics and Biomechanics 7 (2010), 169-176.

[20] S.K. Pandey and D. Tripathi, Peristaltic flow characteristics of maxwell and magnetohydrodynamic fluids in finite channels: Models for oesophageal swallowing, J Biological Systems 18 (2010), 621-647.

[21] A.H. Shapiro, M.Y. Jaffrin and S.L. Weinberg, Peristaltic pumping with long wavelengths at low Reynolds number, $J$ Fluid Mech 35 (1969), 669-675. 

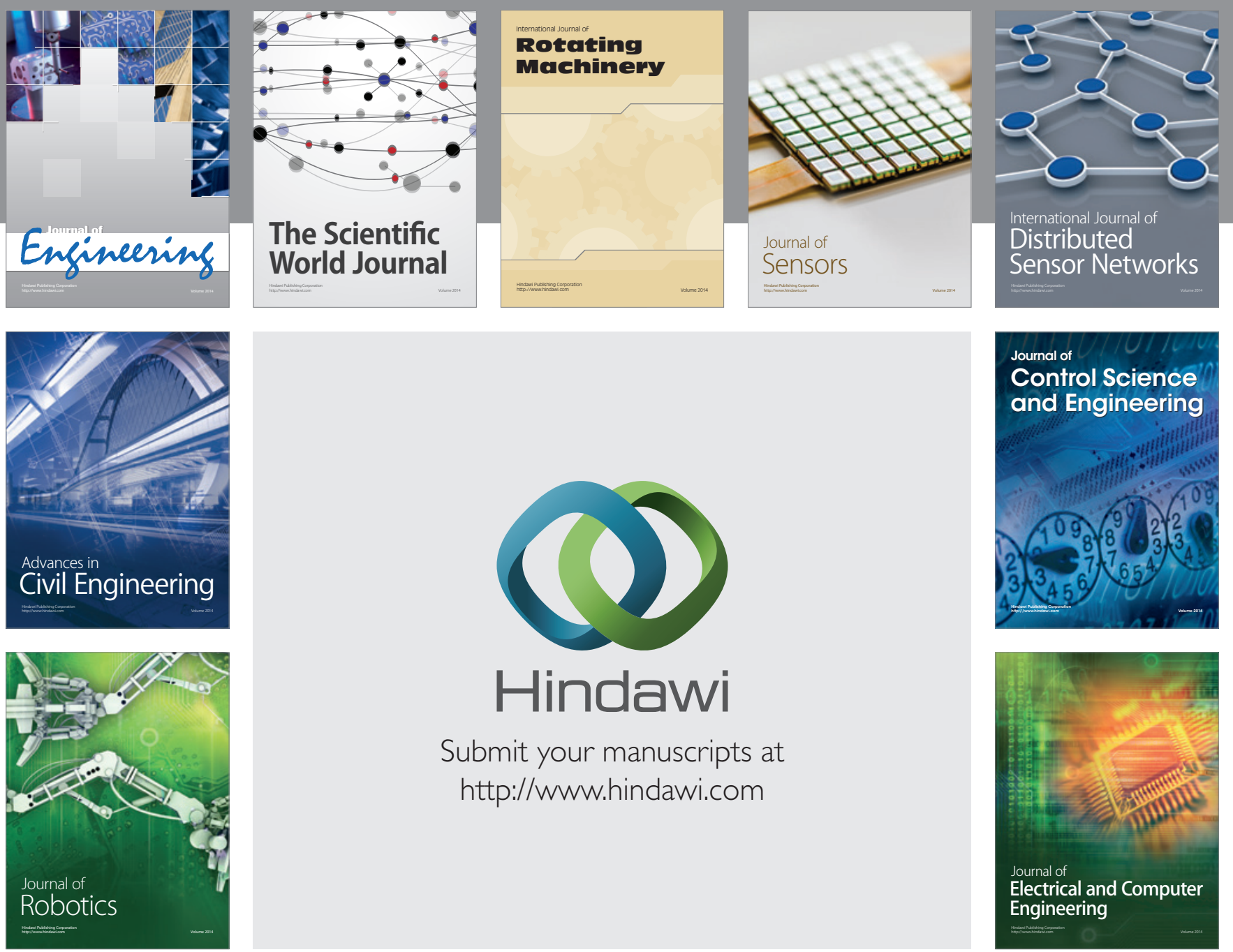

Submit your manuscripts at

http://www.hindawi.com
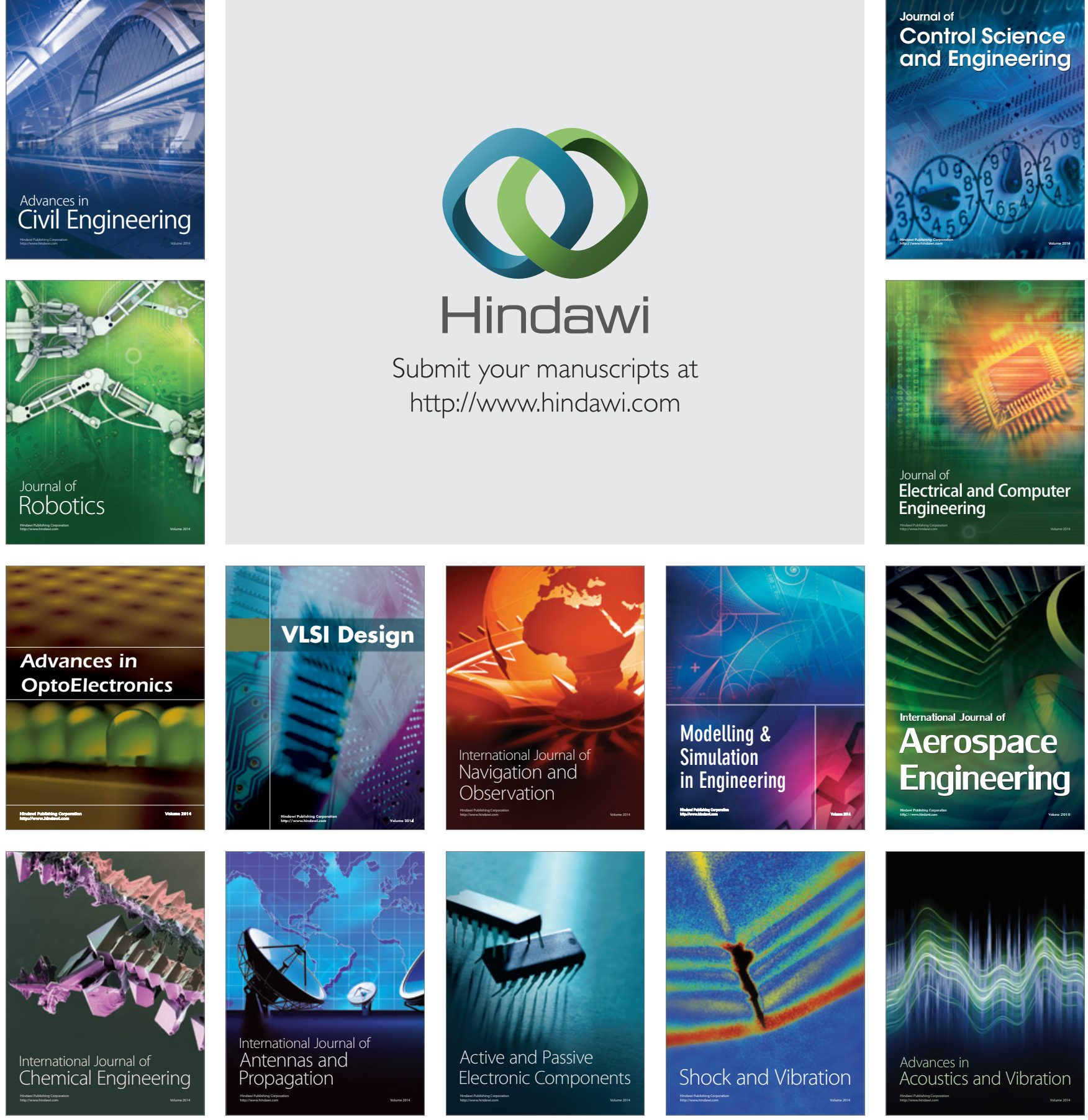